\title{
Evaluación del desempeño pedagógico- didáctico de los instructores en el centro de Diseño, Metrología y Calidad del SENA regional Bogotá
}

\section{Evaluation of Performance Pedagogic and Didactic of the Instructors in Center of Design, Metrology and Quality of the Bogotá Regional SENA}

\author{
Luis Francisco Ochoa Rojas*
}

Fecha de recepción: 30 de enero de 2009

Fecha de aprobación: 27 de febrero de 2009

\section{Resumen}

El presente es un informe de avance del estudio sobre la valoración del desempeño pedagógico didáctico de los instructores del Centro de Diseño, Metrología y Calidad, del Servicio Nacional de Aprendizaje (SENA), Regional Distrito Capital. Dicha valoración es realizada por los aprendices ${ }^{1}$ de los programas desarrollados en el

Magíster en Educación, Universidad de la Sabana (Bogotá-Colombia), Lic. en Psicología-Pedagogía, Universidad Pedagógica Nacional (Bogotá-Colombia), docente de Filosofía de la Ciencia en la Maestría en Educación de la Universidad Santo Tomás (BogotáColombia). Investigador en dicha Maestría en el proyecto de investigación Fundamentación epistemológica de la investigación en educación en Colombia en el siglo XXI. Asesor de proyectos de Investigación en el Programa de Psicología de la Facultad de Medicina de la Universidad del Rosario (Bogotá-Colombia). Asesor metodológico y pedagógico en la metodología de formación por proyectos en el Servicio Nacional de Aprendizaje de Colombia (SENA). Correo electrónico: fochoa@sena.edu.co

1 Se refiere a lo que en otros contextos se denomina estudiante, alumno o discente. 
centro mencionado durante 2007. El propósito del estudio es obtener información de los beneficiarios de la formación profesional integral ${ }^{2}$, para el mejoramiento de los procesos de enseñanza-aprendizaje-evaluación ${ }^{3}$ y para dar elementos de decisión en la contratación de los instructores ${ }^{4}$.

Palabras clave: Desempeño pedagógico-didáctico, proceso de enseñanza-aprendizaje-evaluación, evaluación.

\section{Abstract}

This paper is an advance report of the study on the instructors pedagogical and didactic performance at Sena, Center of Metrology and Quality in Bogotá, D.C. Regional. This evaluation was carried out by the apprentices ${ }^{5}$ from the programs offered by the Center in 2007. The purpose of the study is to gather information from the students who benefit from the professional integral training ${ }^{6}$ to improve the teaching-learning-evaluation processes ${ }^{7}$ as well as provide elements to make decisions on instructors hiring ${ }^{8}$.

Key words: Pedagogical and didactic-performance, teaching-learning-evaluation processes, evaluation.

\section{Introducción}

EI SENA es consciente de la importancia de desarrollar con estrictos criterios de calidad todos los procesos de la entidad. La Dirección General, las Direcciones Regionales, dentro de ellas la del Distrito Capital, y los Centros de Formación, en particular el Centro de Diseño,
Metrología y Calidad deben avanzar sin descanso en el desarrollo de la formación profesional integral, de tal forma que dicho proceso posea el nivel educativo, técnico, tecnológico y productivo que garantice la competitividad de los egresados y de las empresas para enfrentar la exigencia que impone la economía globalizada.

2 Es parte de la educación no formal, que se encarga de la formación técnica y tecnológica de los trabajadores para su inserción en el proceso productivo del país.

\begin{tabular}{ll}
\hline 3 & Es la secuencia de la formación que da el SENA, la cual se explicará en detalle más adelante. \\
\hline 4 & Se refiere a lo que en otros contextos se denomina docente, maestro o profesor. \\
\hline 5 & It refers to students in other contexts. \\
\hline 6 & It has to do with the informal education that \\
\hline 7 & It is SENA training sequence, which will be explained in the paper. \\
\hline 8 & It refers to teachers in other contexts.
\end{tabular}




\section{Justificación}

El Centro de Diseño, Metrología y Calidad, en la formulación de sus proyectos de investigación y asesoría pedagógico-didáctica asistida, ha contemplado como componente fundamental de éstos, entre otros, realizar un estudio sobre el desempeño pedagógico-didáctico de los instructores dentro del proceso formativo, toda vez que este proceso constituye un elemento esencial de las actividades propias de la formación profesional; estudio que tiene como propósito descubrir aspectos en los que se requiere perfeccionar la práctica pedagógicodidáctica de los instructores dentro del proceso de enseñanza-aprendizaje-evaluación de los aprendices.

Como consecuencia de lo anterior, se estructura el estudio denominado Evaluación del desempeño pedagógico-didáctico de los instructores en el Centro de Diseño, Metrología y Calidad del SENA Regional Distrito Capital.

\section{Problema de investigación}

En el desarrollo de los programas de formación del nuevo talento humano para el aparato productivo de Colombia se hace necesario que la productividad y la competitividad de los trabajadores se refleje en su desempeño dentro de las empresas. Teniendo en cuenta esta premisa, se parte de la hipótesis de que la excelencia de la formación conduce a la excelencia en el desempeño laboral de los egresados. Esta relación se hace concreta en el mejoramiento continuo de los desempeños pedagógico-didácticos de los instructores en su labor formadora en las aulas, talleres y laboratorios del SENA, en general y del Centro de Diseño, Metrología y Calidad, en particular. Por tanto, se requiere construir una serie histórica de información que determine la percepción de los aprendices sobre los desempeños de los instructores. Ello servirá para ubicar las fortalezas y las debilidades en el trabajo pedagógico-didáctico de la formación profesional que imparte el Centro mencionado.

Como consecuencia de lo anterior, a continuación, se plantea el problema de investigación a través de la siguiente pregunta: ¿en qué medida valoran los aprendices el desempeño pedagógico-didáctico de los instructores en el Centro de Diseño, Metrología y Calidad del SENA Regional Distrito Capital en el año 2007?

\section{Marco teórico}

Para los efectos de este proyecto, se hace una primera aproximación teórica que deberá ser ampliada en el informe final de investigación, una vez se haya realizado todo el trabajo de campo, las tabulaciones, los análisis de datos, la interpretación correspondiente, las conclusiones y las recomendaciones. También se anexan algunas definiciones que harán las veces de marco conceptual para los efectos del estudio.

En las últimas décadas se han realizado varios esfuerzos con el fin de aclarar el significado de la evaluación y, al mismo tiempo, distinguirla de otros conceptos como el de medición y el de investigación. Como ya se dijo, en la literatura internacional hay varios estudios que traba- 
jan la conceptualización de la evaluación, los cuales han dado como resultado el establecimiento de varios enfoques evaluativos con su correspondiente significado en educación y en las ciencias sociales.

Muchos de esos enfoques se les ha llamado "modelo", cosa indebida, porque el término tiene una significación específica en las ciencias formales como interpretación de una teoría abstracta, y en las ciencias factuales, la palabra modelo se refiere a un sistema teórico que se manifiesta como un conjunto de proposiciones relacionadas entre sí, lógica y epistemológicamente.

Habiendo hecho esta precisión, se puede decir que se conocen "modelos" de evaluación como el CIPP, el modelo de Discrepancia y el modelo de Evaluación Libre de Fines. Stake (1981), citado por Nevo (1983) sugirió que éstos se podían entender más como interpretaciones que como modelos (Franco \& Ochoa, 1997, p. 63).

Por otra parte, se han tratado de organizar clasificaciones con la gran cantidad de trabajos en la literatura internacional de la evaluación.

Tales clasificaciones (Guba \& Lincoln, 1981; House, 1980; Popham, 1975; Stake, 1976; Stufflebeam y Webster, 1980; Worthen y Sanders, 1973) son una contribución significativa, mediante revisiones críticas de la literatura en evaluación, pues denotan las similitudes y diferencias entre los diversos enfoques (Nevo, 1983, p. 1).

Con respecto a la definición de evaluación hay muchos trabajos y propuestas, por ejemplo,
Tyler (1950) la concibe como un proceso para determinar hasta qué punto se han logrado los objetivos educativos. Nevo (1983) señala que:

[...] otra definición muy aceptada es aquella de "suministrar información para tomar decisiones", sugerida por varios evaluadores tales como Cronbach (1963); Stufflebeam (Stufflebeam et al., 1971) y Alkin (1969). Recientemente, se ha alcanzado un amplio consenso entre evaluadores con respecto a la definición de evaluación como la determinación del mérito o el valor (Eisner, 1979; Glass, 1969; House, 1980; Scriven, 1967; Stufflebeam, 1974); o como actividad que comprende tanto la descripción como el juicio (Guba \& Lincoln, 1981; Stake, 1967, p. 1).

Por su parte, Stufflebeam (1987) presenta la definición que ha adoptado el Joint Committee on Standars for Educational Evaluation. La definición es la siguiente: "la evaluación es el enjuiciamiento sistemático de la valía o el mérito de un objeto" (p. 19).

En el caso de América Latina las concepciones sobre la evaluación están íntimamente ligadas con los enfoques pedagógicos, esto significa que la evaluación está circunscrita básicamente a los procesos educativos. Sólo en los últimos tiempos, por la influencia norteamericana, se ha ampliado su visión a otras áreas de las ciencias sociales.

Los conceptos sobre evaluación educativa han venido evolucionando paralelamente a los cambios que ha sufrido la concepción misma de la educación. No obstante, la educación centrada en la transmisión de conocimientos, estudio y desarrollo de 
contenidos temáticos, unida a prácticas evaluativas en donde éstas se identifican con notas, exámenes escolares y con la categoría de "aprobado", "no aprobado", tiene una fuerte tradición en el hecho de querer reducir la función de la evaluación a la asignación de calificaciones y a la promoción (Equipo Investigador, 1987, p. 6).

Murcia Florián (1991) dice que "tanto el problema de repitencia como el de deserción y el de analfabetismo, en general, están estrechamente relacionados con la problemática de la evaluación" (p. 91).

Díaz (1988), citado por Murcia Florián (1991), señala que el término "evaluación" no tiene tradición dentro de los planteamientos pedagógicos: tampoco se puede considerar sólo como un reemplazo "científico" de las prácticas de examen; en el fondo es una concepción que ha surgido con el proceso de industrialización. A través de estos modelos y teorías se concretan las exigencias de control individual y social que tales procesos exigen, por ello responden a una pedagogía para la industrialización. Su génesis se vuelca dentro de una visión funcionalista y una concepción conductual del ser humano (pp. 91-92).

En Latinoamérica se pueden distinguir dos grandes enfoques que Martinic y Walker (1987) denominan tradicional y alternativo. "Por enfoque tradicional se entiende aquella perspectiva donde la evaluación se refiere a la medición del grado en que los objetivos son alcanzados" ( $p$. 5). Este enfoque tiene su origen en la concepción tyleriana, la cual ha sido arduamente criticada, pues no da cuenta cabal de los procesos educativos, así como de su fundamentación epistemológica está emparentada con el objetivismo y la neutralidad valorativa, defendidos por el empirismo y el positivismo lógico. Otros elementos de crítica a este enfoque apuntan a lo inadecuado de muchas de las mediciones, a la rigidez e inadecuación de los modelos frente a la dinámica y la complejidad de los contextos populares y a la no participación de los beneficiarios en el proceso de evaluación de los programas sociales y educativos.

Como reacción a la concepción tradicional de evaluación, con sus variados matices y aplicaciones, han surgido alternativas que adoptan distintos nombres, varias de las cuales fueron presentadas anteriormente como nuevos enfoques de evaluación y enfoques participativos de evaluación.

\footnotetext{
Entre éstas destacamos a la evaluación iluminativa, evaluación sensible, autoevaluación, evaluación participativa. Se mira los programas desde su contexto y al interesarse en las significaciones se prioriza en los métodos cualitativos, en la formación de carácter subjetivo y en instrumentos que promueven la autoevaluación (Martinic \& Walker, 1987, pp. 14-15).
}

Ya se ha recorrido cierto camino por estos "enfoques alternativos" de evaluación. Se ha avanzado en la construcción teórica y las evidencias empíricas son abundantes y ricas. A pesar de lo anterior, permanecen ciertos problemas en relación con el poco rigor con que se abordan algunas dimensiones de las experiencias de trabajo; aún no hay conexión teórica con los planteamientos de otras concepciones ni hay 
un lenguaje técnico-científico suficientemente legitimado que sea reconocido por las instancias que toman decisiones en este campo.

Murcia Florián (1991) dice que es necesario que se incremente el debate entre los enfoques tradicionales y alternativos y que:

[...] para enriquecer el debate y llegar a una producción de conocimiento, es preciso consultar diversos autores e investigadores, tanto desde los paradigmas teóricos y metodológicos como desde el punto de vista interdisciplinario del objeto o contenido de evaluación [...] Asistimos, actualmente, a un desplazamiento del objeto de la evaluación: de objetivos y productos a procesos y sentidos (p. 94).

De lo anterior se desprende una inmensa vena de acciones de investigación que facilita la participación de los distintos actores de los procesos educativos y sociales, que permitan la construcción de conocimiento desde la cotidianidad con nuevas perspectivas; así como el uso de ese conocimiento en procesos dialógicos de convergencia en la educación y en la cultura, en la cual la evaluación pueda tener un papel distinto del que ha tenido hasta ahora.

Visto lo anterior se puede decir que no existe aún unidad de criterios teóricos ni epistemológicos que permitan construir una definición de evaluación que satisfaga las diversas perspectivas. Parece que esta pluralidad de conceptos y enfoques existe justamente por la intencionalidad diversa que tienen los agentes de la evaluación en su acción misma; tal como lo señala en otras palabras la cita de Murcia
Florián (1991), la cual dice que: "al cambiar el concepto y el sentido institucional cambia el concepto y el sentido de la evaluación para una comprensión más profunda" (p. 91). También es válido lo que dicho autor afirma sobre el carácter cambiante del concepto de evaluación, según el papel que se le asigne en la práctica evaluativa. A pesar de lo anterior existe un elemento común en todos los enfoques evaluativos; se trata de que en cualquier enfoque de evaluación siempre está presente una acción intencional que busca determinar el valor o el mérito del objeto que se va a evaluar.

Cuando se habla de acción intencional, se hace referencia a la acción en los términos definidos desde Habermas, la cual, cuando se refiere a la evaluación posee la pretensión anticipada de juzgar la valía o el mérito del objeto por evaluar.

Los términos valía o mérito también deben ser definidos para enriquecer nuestro concepto de evaluación. Scriven (1981) ya ha indicado las trascendentales diferencias que hay entre ellos y el importante papel que desempeñan a la hora de determinar el valor de algo. Por ejemplo, un programa para formar profesores de Historia, ¿podrá producir profesores que enseñen Historia a otros de un modo efectivo y de manera que se pueda confiar con ellos? En general, ¿hará bien lo que se supone que debe hacer? Si lo hace, será meritorio, pero puede que no sea válido. Por ejemplo, si la sociedad ya no necesita más profesores de Historia porque ya tiene más de los que puede emplear, entonces aunque el programa sea muy bueno -y quizá sea mejor que otros parecidos-, continuar con el mismo nivel de expansión del programa quizá no sea la mejor manera de gastar sus escasos recursos. Aunque un programa meritorio 
puede no ser válido, debemos indicar que un programa que no sea meritorio no puede ser válido. Puede que no sea bueno formar malos profesores de Historia aunque exista una gran escasez de ello (Stufflebeam \& Shinkfield, 1987, pp. 20-21).

De acuerdo con lo anterior, para el presente estudio se ha optado por concebir en general la evaluación, como: la acción intencional en forma de juicio, determinada con anticipación, que pretende ponderar la valía o el mérito de un objeto evaluable.

Por otra parte, el desempeño pedagógico didáctico de los instructores del SENA, como objeto de evaluación por parte de los aprendices, se concibe como el conjunto de conductas que son deseables, por parte de los instructores, para facilitar el proceso de enseñanzaaprendizaje-evaluación. Dicho proceso es la columna vertebral de la formación profesional integral, que desarrolla esta institución en la generación de talento humano para el sector productivo del país.
La enseñanza recoge aquellos comportamientos del instructor en los cuales utiliza sus conocimientos, habilidades y destrezas en la orientación del aprendizaje de las áreas técnico-productivas. Por su parte, el aprendizaje se concibe de acuerdo con las teorías cognitivoconductuales, en las cuales la comprensión de los nuevos saberes se hace contextualizándolos con el aparato de aprendizajes previos que trae el sujeto; y los dominios de esos nuevos saberes se manifiestan como desempeños de los aprendices en los que saben el significado del saber; saben usar el saber generando productos, saben desempeñarse con otros como seres sociales e individuales.

La evaluación es una consecuencia de los dos elementos anteriores, puesto que se valoran los desempeños de los aprendices con respecto a unas normas de competencia productiva en las distintas áreas técnicas. El desempeño pedagógico-didáctico se compone de los siguientes elementos para los efectos de la presente investigación:

Tabla 1.

\begin{tabular}{|l|l|}
\hline $\begin{array}{c}\text { Elementos } \\
\text { componentes } \\
\text { del desempeño } \\
\text { pedagógico didáctico }\end{array}$ & Comportamientos que definen operacionalmente cada componente \\
\hline $\begin{array}{l}\text { 1. Cumplimiento y uso } \\
\text { eficiente del tiempo }\end{array}$ & $\begin{array}{l}1.1 \text { Cumple con el tiempo programado para la orientación del módulo. } \\
1.3 \text { Cumple con el trabajo concertado con los aprendices. } \\
1.4 \text { Desarrolla lo establecido en el plan de formación. }\end{array}$ \\
\hline
\end{tabular}




\begin{tabular}{|c|c|}
\hline $\begin{array}{l}\text { 2. Conocimientos } \\
\text { técnicos. }\end{array}$ & $\begin{array}{l}\text { 2.1 Muestra dominio técnico en el desarrollo de las actividades pro- } \\
\text { gramadas. } \\
\text { 2.2 Argumenta sus explicaciones sobre los temas y su posición frente } \\
\text { a ellos. } \\
2.3 \text { Maneja referencias de material educativo, bibliografía, Internet y } \\
\text { otras fuentes de información para la orientación de los aprendices. } \\
\text { 2.4 Se preocupa por innovar los conocimientos de su área, a través } \\
\text { de experiencias compartidas con los aprendices. } \\
2.5 \text { Contextualiza los contenidos del módulo con las características y } \\
\text { problemas del medio (social, científico, tecnológico, académico, etc.). }\end{array}$ \\
\hline $\begin{array}{l}\text { 3. Fomento del } \\
\text { espíritu investigativo }\end{array}$ & $\begin{array}{l}\text { 3.1 Fomenta la crítica y el debate académico como parte del desarrollo } \\
\text { de las acciones de formación. } \\
\text { 3.2 Tiene en cuenta y respeta los saberes y opiniones de los apren- } \\
\text { dices. } \\
\text { 3.3 Promueve nuevas actitudes y posiciones académicas frente a los } \\
\text { diferentes saberes. } \\
\text { 3.4 Facilita la comunicación y el diálogo en la solución de problemas } \\
\text { académicos o investigativos. } \\
\text { 3.5 Incentiva y asesora al aprendiz en el análisis crítico de diversas } \\
\text { fuentes de información (artículos, textos, Internet). } \\
\text { 3.6 Fomenta el trabajo en equipo. } \\
\text { 3.7 Promueve el desarrollo de habilidades comunicativas tanto orales } \\
\text { como escritas. } \\
\text { 3.8 Toma situaciones o problemas del medio (social, científico, tec- } \\
\text { nológico, académico, etc., según el caso) y los convierte en objeto de } \\
\text { aprendizaje. }\end{array}$ \\
\hline $\begin{array}{l}\text { 4. Metodología de } \\
\text { enseñanza (didáctica) }\end{array}$ & $\begin{array}{l}\text { 4.1 Hace un adecuado uso de los recursos didácticos. } \\
\text { 4.2 Utiliza estrategias didácticas que facilitan la apropiación de cono- } \\
\text { cimientos (estudio de casos, proyectos, simulaciones, juego de roles, } \\
\text { etc.). } \\
\text { 4.3 Es creativo y dinámico en el desarrollo de las actividades. } \\
\text { 4.4 Genera interés y motivación por los temas del módulo. } \\
\text { 4.5 Promueve la participación activa de los aprendices. } \\
\text { 4.6 Promueve la formación de valores. } \\
\text { 4.7 Promueve el autoaprendizaje. }\end{array}$ \\
\hline
\end{tabular}




\begin{tabular}{|c|c|}
\hline $\begin{array}{l}\text { 5. Evaluación aplicada } \\
\text { a los aprendices }\end{array}$ & $\begin{array}{l}\text { 5.1 Respeta los acuerdos sobre procesos y modalidades de evaluación } \\
\text { concertados. } \\
\text { 5.2 Cumple con las fechas de evaluaciones acordadas con los apren- } \\
\text { dices. } \\
\text { 5.3 Realiza evaluaciones coherentes con los contenidos, actividades } \\
\text { y metodologías desarrolladas en el módulo. } \\
\text { 5.4 Realiza una evaluación permanente. } \\
\text { 5.5 Es objetivo en las evaluaciones. } \\
\text { 5.6 Entrega de manera oportuna los resultados de las evaluaciones. } \\
\text { 5.7 Acompaña las evaluaciones con recomendaciones formativas. } \\
\text { 5.8 Atiende y resuelve oportunamente los reclamos de los aprendices } \\
\text { sobre sus evaluaciones. }\end{array}$ \\
\hline & $\begin{array}{l}\text { 6.1 Trata a los aprendices con equidad y respeto. } \\
\text { 6.2 Asume un comportamiento ético acorde con su dignidad de ins- } \\
\text { tructor. } \\
\text { 6.3 Promueve espacios de discusión y profundización sobre temas de } \\
\text { importancia académica o del Centro. } \\
\text { 6.4 Demuestra sentido de pertenencia hacia la institución. } \\
\text { 6.5 Muestra respeto por las posiciones teóricas, conceptuales o me- } \\
\text { todológicas diferentes a la suya. } \\
\text { 6.6 Demuestra respeto por la persona o por los planteamientos de } \\
\text { sus colegas. } \\
\text { 6.7 Se muestra receptivo a las críticas y sugerencias planteadas por } \\
\text { los aprendices. }\end{array}$ \\
\hline $\begin{array}{l}\text { 7. Valoración general } \\
\text { del instructor }\end{array}$ & $\begin{array}{l}7.1 \text { ¿Con este instructor usted superó el } 70 \% \text { del nivel aprendizaje } \\
\text { esperado? } \\
7.2 \text { ¿Usted cursaría otro módulo con este mismo instructor? }\end{array}$ \\
\hline
\end{tabular}

Por todo lo anterior, para este estudio, la evaluación de los aprendices sobre el desempeño pedagógico-didáctico de los instructores del Centro de Diseño, Metrología y Calidad, es la ac- ción intencional de los aprendices en forma de juicio, determinada con anticipación, que pretende ponderar la valía o el mérito de los desempeños pedagógico-didácticos de los instructores. 